\title{
準備委員会企画招待講演
}

\section{Entering a Symbolic World: Cognitive Development in the Pre-literacy Years}

\author{
Katherine Nelson, Ph.D.
}

(Distinguished Professor of Psychology Emerita, City University of New York Graduate Center)

My talk today focuses on how children learn and use language during the preschool years for both cognitive and communicative purposes. My approach to these issues reflects a cultural-historical neo-Vygotskian view of child development.

It is during the early childhood years that children first gain entry into the symbolic cultural world, which I have conceptualized as entering the Community of Minds. To begin to interpret the symbolic world the child must begin the process of entering into the Community of Minds, that is the cultural community wherein people share their ideas and perspectives on the world, primarily through linguistic means. The child's entry is made possible via the joint contributions of the biology which underlies experience, the cultural symbols themselves, symbolic language that eventually reveals much about the mind and world, and the higher levels of cognition made possible by symbolic representations.

\section{Infancy in the Symbolic World}

All children are born into and develop within a specific cultural milieu, where meanings are embedded in symbols. Among the cultural artifacts and symbols that surround children from the beginning of life are multiple uses of linguistic forms-songs, stories, and protoconversations. Parents around the world invoke for themselves cultural narratives about how children should develop, behave, believe, and grow up within an idealized world. In the anthropologist Crapanzano's (1990) view the dyadic exchange between mother and child always includes a silent but ever-present "third voice," the voice of culture speaking through the parental discourse.

The important claim here is that the infant's mind is not shareable, and that others cannot share their thoughts with the infant. Infants and young children easily express their emotions, and adults have little difficulty in reading these, or in responding to the infants expression of needs and wants. However, to the extent that infants form concepts, generate novel ideas, engage in reasoning, these processes are closed off to the view of other people. In consequence, the main tasks of the period between birth and school entry - the period that I am calling "pre-literacy"-are concerned with the process of becoming conscious of and mastering the world of symbols through which the private mind becomes extended into the cultural world.

The conventional symbolic system of language produces profound differences in thought that differentiate humans from other primates, as they also differentiate children from their former infant selves. The impact of language with respect to these differences is, I think, greatly underestimated in most current developmental theories. There is a tendency among professionals as well as parents to take what children say as simply expressing what they already "know" or "think." This assumption has led some eminent theorists to adopt the view that infant thinking is no different from that of adults-it's just that they cannot express it properly. When acquired, language then is taken to be a communicative tool but not in any important way a cognitive tool. It is seen as a mode of learning from others but not as a mode of thinking for oneself. The communicative-only view is tied to a brand of cognitive nativism and universalism that tends to deny significant differences in thought resulting from language or culture whether in development or over evolutionary and historical time. In contrast, the Vygotskian view sees the development of thought as both historically and culturally contexted, and thereby reliant on language, with little place for either nativism or universalism. These contrasting views relate to contrasting models of how children learn the meaning of words. 


\section{Forms and Functions of Word Learning}

In the mapping model the problem for the child is framed as that of acquiring a pre-defined and narrowly bounded meaning of a word-its correct reference - determined by its place in lexical semantics. The assumption is that the problem is to map the word onto a pre-existing concept. What is in question is the accuracy of the child in attaining correct reference. It seems to follow automatically that thought -i.e., concepts - is both independent of and prior to language, a relation that is represented in the classic semiotic triangle displaying the relation of word, object, and concept.

Different disciplines interpret the triangular relationship in different ways. In the developmental interpretation of the claim that concepts precede words it is asserted that children must have concepts in order to match words with them. This claim seems to hold for many first words as I in fact suggested many years ago. The alternative interpretation goes beyond this to emphasize that words themselves shape concepts. As I also pointed out many years ago children's concepts might be quite different from the adult's meaning for a word, and different in interesting ways, revealing something about children's pre-verbal thought. Based on their experiences during the first year, which include socially shared activities such as eating, bathing, going for a walk, visiting friends, and so on, children build up knowledge of the things and events of their world from a unique child-based perspective. Thus children come to word learning with concepts and categories based on their own direct experience of the world, and use these as a bootstrap toward the interpretation of words that are used in their daily lives. For example, their concept of "dog" may include many functional and perceptual characteristics, and may overlap only somewhat with the concept that an adult has of the category. Nonetheless, when the child learns to say "dah" in the context of dogs, the two will be able to share the same label for instances of dog in books and on the street. In addition, as is often observed, a 1-year-old may extend the label to other 4-legged creatures, indicating either that the category is not bounded in the same way that the adult's is, or that the uses of words to bind categories is not yet well-understood. Although the prob- lem of differences in concepts tends to be set aside by most word learning theorists today as uninteresting or irrelevant, I think it is central to our understanding of the relation of words and concepts as well as to questions of how children learn the meaning of words, and how words function for them.

The problem is that adults use words to communicate their concepts; the child's problem then is to match-or construct-a concept that fits the context of the adult's use. In ordinary communicative contexts the child may have available a concept that seems to fit the adult's use of a new word, or she may not. If not, the child's burden is to construct, on the basis of evidence from current context, ongoing discourse, and general background knowledge, a meaning that fits the adult's use. This is likely to be the first time in which the child is confronted with the problem of sharing meaning with another through the use of symbols.

Unless it is assumed that child and adult see the world in the same way and have the same store of concepts to be named, there is a problem of aligning different concepts to the same words. The extended semiotic triangle exemplifies this essentially socialcultural problem of meaning matching. This problem is not restricted to first words of course, but extends to word learning throughout life, including classroom learning and learning words through reading.

It is important to emphasize that meaning matching is not the same as mapping words to pre-existing concepts, the pervading model of lexical acquisition based on the idea of mapping between a well-defined system of linguistic meanings and a set of cognitive concepts that are assumed to be held by people in general. From the perspective of meaning matching word learning is an extension of the social communicative relation that has been set up throughout infancy. Infants begin to learn the words because the words are part of the interaction process. It is not that infants are programmed to learn the right words to go with the right concepts; that is to say that word learning is not a process of truth and error; rather it is a pragmatic process of making words work in contexts of social interaction.

It is clear that in the classical conception of the 
naming game the burden of learning is put on the child; the tutor's role is simply to point and name. However, when we acknowledge that word learning is (at least) a two-person game, with more than one round, taking place within a situation of shared action and understanding, its ultimate communicative success is less mysterious. Not only is the object one of shared meaning, but the game played in achieving it involves shared roles and responsibilities.

Even from the beginning of learning children do not focus only on objects. Fewer than half of the words that children acquire even in the first year of learning (up to about 300 words) are names of concrete objects (basic level object categories or BLOCs). Many are proper nouns, names of individuals, places, brand names, and so on. Others are terms for substances such as water, sand, glue, shampoo, and so on. These can also be pointed to, but the inferences to be drawn with regard to what is named are quite different from either the proper or common name situation. More problematic are what I term XBLOCs, nouns that stand for cultural constructs such as temporally organized events like "lunch" or "party", or places such as "park" or "basement", or role designations such as "brother" or "doctor." Still other nouns designate natural phenomena like "wind" "noise" "snow" or "storm." And some early learned nouns are superordinate category terms like "animal" and "food." Moreover, some early words act as both noun and verb in the same form, such as "drink" "kiss" and "walk." Thus the "naming game" paradigm must fail for even for most of the words that children begin using and for all of the more abstract terms they learn in the preschool and later years.

It is widely noted that children acquire something on the order of 5 to 10 new words each day between the ages of 2 and 6 years. What is less widely noted is that the same rate continues at least through the years of formal schooling, that is through high school for US students, eventuating in total vocabularies of between 50,000 and 100,000 words. Obviously not all of these words are names of objects, and indeed many are words referring to abstract concepts like temporality and causality, or terms for mental states such as "know" and "think" or theoretical terms such as superordinate category labels or scientific constructs.
A corollary to the assumptions of the naming game is that if the game gets off on the right foot, then words that refer to other things than objects e.g., actions (verbs), or attributes of things (adjectives) will be readily learned in analogy to the thing world, or to fill out the kinds of statements that people and children might want to make about things. But this doesn't work for abstract words of this kind.

The mapping model relies on the child's non-verbal expansion of concepts and a proposal of a "fast mapping" process, to account for how word learning in the pre-school period can be "fast, easy, accurate and efficient." In contrast, the meaning matching, social game model, rejects this model as inadequate to the joint problem of concept and word learning in the preschool and later in the school years. Rather, we propose that the same process that applies in early childhood word learning, that is, meaning matching, applies to other kinds of words, including abstract constructs. This is not to say that children are fast, accurate and efficient in this process. Indeed, the evidence is clear that they are none of these, when we focus on the meanings of all kinds of words, rather than on the simple learning of word forms or names of things. Thus we call on the ideas of learning from use, which echoes the ideas of the philosopher Ludwig Wittgenstein. Studies of the use of words by preschoolers shows that the acquisition process is dependent upon extensive practice in which parental use provides a model in specific contexts of how the word is used, although it does not provide a specification of what the word means.

The kernel of truth in the original naming game lies in the "game" part, and when we turn to the different conception of language games put forth by Wittgenstein (1953), we find a better fit to the child's situation. Wittgenstein also began with a kind of naming game, but one that took place in joint activities where naming was functional within the activity and its use indicated its signification. From Wittgenstein we can take two lessons relevant to children's acquisition of the meaning of words: that they are learned in the course of activities where words are used by others in functional contexts; and that words do not have definite single meanings, based on reference, but rather meanings that are derived from, and are displayed in, 
use in functional discourse contexts. Thus children must become sensitive both to the language uses of the community and to the implied conceptual bases of language in order to be understood as well as to understand. That is, they must become adept at playing Wittgenstein's language games.

\section{Beyond Words to Representational Language}

By using language the child enters into a social/ cultural world that extends beyond her own experience and beyond the family into the society at large. The words she learns and uses are used in the same conventional ways by all others who share the same cultural/linguistic world. The child's uses of words are constrained by the uses of these others; but by the same token, they take her into a "social network of minds."

Implicit in the idea that meaning is learned from use is that children's understanding and knowledge of the world expands with their exposure to language, as they participate in conversations about aspects of the world that they have not yet and may never experience directly and personally, including aspects that are imaginary constructions of the culture at large. By about 4 years of age children have in place a symbolic system of meanings-grammatical and semantic - that can be used to formulate new representations of the real or imagined world to be shared with other people. The representational function of language, internal to self or external for sharing with others, is what language is all about. It communicates information; it conceptualizes; it structures narratives; it hypothesizes theories.

When two or more persons are conversing the representation they are jointly constructing is external to each. Each contributes but the conversation as such is not confined to the "inside" of either individual. It is thus necessary for the listener to translate what is verbally conveyed from language to nonverbal thought; this is what the child must become competent at as proficient language is achieved. But this possibility is a developmental achievement, making it possible for the individual to formulate a linguistic representation of a state of the world (real or imagined) internally (in the mind through private or inner speech) and to use that representation as an object to be remem- bered or to be subjected to further thought. There cannot be a linguistic representational cognitive system in place until the child has acquired sufficient language to "think in" language. This requirement is easily understood in terms of a second language, where as most learners know, it takes a great deal of learning and use before becoming capable of "thinking in" the second language. Many of us never achieve this for more than one language.

How then do children interpret language before becoming capable of thinking in it? For example, when a parent reads a story to a child the representation is external to the child, on the page and in the voice of the parent. What then about the individual's representation, for example the child's representation of the story?

Similarly to word meaning the mediational model presented in Nelson (1996) emphasized that understanding extended text or discourse requires extensive practice with language in use for functions such as narrative, description, and explanation. The process involves retention of ongoing spoken language over extended time segments as each bit is related to the next, or held to be connected to something that comes later in the sequence. The mental representation in language provides support for the retention of the meaning of the story. However, the model also implies that becoming a good story understander rests on the experience of listening to stories and engaging in conversations that go beyond the here and now. Practice in using representational language, especially in narrative form, brings out the relations of time and space, other persons and other minds, representing the past and the future as well as possible worlds. Thus it advances the child into the cultural symbolic world. The basic language functions of establishing shared conceptual meanings and representations within a cultural community has important implications for understanding the process of how language gets into the mind and establishes conceptual systems that are not derived from observables in the real world.

\section{The Community of Minds}

Two essential accomplishments of the pre-literacy period have been highlighted: acquiring shared concepts through conventional words and constructing 
representations in and through language. A byproduct of these achievements is the movement into the larger community of minds that exists in the social-cultural world where the child is beginning to take a place. Members of the community depend upon the existence of shared symbolic systems, and use these to take the perspective of others within the community. Instead of accepting the terminology of "theory of mind" I emphasize the entry into this larger community where disparate views may be encountered but also understood through shared language and meanings. These are not individual accomplishments, but ones that are achieved only through the collaboration of adults with children and through practice in acquiring meanings in context.

The story here has been a brief one, focused perhaps too exclusively on the topic of word meanings when the ultimate point is about understanding worlds of meaning.

\section{References}

Nelson, K. 1996 Language in cognitive development: The emergence of the mediated mind. New York: Cambridge University Press.

Wittgenstein, L. 1953 Philosophical investigations. New York: Macmillan. 